\title{
Influence of Fatigue Loading on the Residual Stress Distribution in Prestressing Steel Wires
}

\author{
Jesús Toribio, Miguel Lorenzo and Diego Vergara \\ Department of Materials Engineering, University of Salamanca, E.P.S., Campus Viriato, Avda. Requejo 33, 49022 Zamora, Spain
}

\begin{abstract}
This paper analyzes the influence of fatigue loading on the residual stress profile in high strength steel wires. To this end, different sinusoidal loads with diverse values of maximum loading level and number of cycles were simulated on wires in which several residual stress profiles had been previously introduced, some of them with a tensile state and others with a compressive state. An analysis was made of the evolution with time of such residual stress laws by comparing them at key instants of loading, that is, at initial instant, at maximum load, at minimum load and at final instant. Numerical results show only a minor influence of fatigue loading on the residual stress profile.
\end{abstract}

Key words: Prestressing steel wires, cold drawing, fatigue, residual stress redistribution.

\section{Introduction}

The drawing process applied in the manufacture of prestressing steel wire (widely used in structural elements) usually produces residual stresses in the material [1, 2], even without any external loading or thermal gradients applied on the wire. The profile of these stress states can present different configurations [3], but in all of them the maximum value of stress is placed at the external surface of the wire. The evolution of the residual stress is decreasing with depth until reaching a value after which it may be assumed as constant throughout the internal section of the wire. Common values at which residual stresses become zero are usually about a tenth of millimetre [3].

Residual stresses play an important role in the process of initiation of surface cracks. It is well known that the industrial use of different surface treatments, e.g., shot peening, provides compressive residual stress at the material surface, thus delaying the initiation and propagation of cracks [4]. For these reasons it is essential to know the evolution of the residual stress

Corresponding author: Jesús Toribio, Civil Engineer, MSc, PhD, professor, research fields: fracture of metals (pearlitic and austenitic steels), stress corrosion cracking, hydrogen embrittlement. E-mail: toribio@usal.es. profile with the parameters characterizing the fatigue loading, such as the number of cycles and the maximum stress level, since these parameters are directly related with initiation and growth of cracks [4].

The residual stress profiles appearing in a prestressing steel wire can be different in two aspects: the surface stress level and the depth where the residual stresses become null. In order to idealize these profiles (observed experimentally by the application of diverse techniques such as Neutron or X ray diffraction [5]) in this paper a linear variation of residual stresses is assumed from the surface until the point from which the stress remains constant. This approach allows one to reproduce the general shape of the 0 .

Residual stresses profiles observed with commonly used measurement techniques without any loss of generality.

This paper deals with an analysis of the influence of fatigue loading on the residual stress profile in high strength steel wires that are typically used in construction. The data obtained in this study may be relevant in the research of hydrogen transport phenomena through diffusion within the material, producing hydrogen embrittlement of the component [6]. These phenomena are potentially very dangerous, 
mainly in structural elements designed in engineering $[7,8]$. Thus in this study the evolution of mean normal (or hydrostatic) stress, a variable representing the stress state of the materials according to the model of hydrogen diffusion assisted by stresses and strains [9-11], is obtained in each step of the fatigue loading process. From these numerical results, it is possible to know the points where the hydrogen is potentially accumulated, i.e., the places where the micro damage might appear inside the material.

\section{Numerical Modeling}

In this study, four fatigue loading schemes were considered, as shown in Fig. 1. All the schemes consisted of sinusoidal cycles oscillating between a null stress and a maximum stress of 1000 and 1200 MPa (i.e., about $75 \%$ and $90 \%$ of $\sigma_{Y}=1300 \mathrm{MPa}$, being $\sigma_{\mathrm{Y}}$ the $0 \%$ offset yield strength of a typical prestressing steel used in construction). To include the effect of the number of cycles in this analysis two loading sequences of 10 and 20 cycles were considered. The type I illustrated in Fig. 1 represents a fatigue loading scheme of 10 cycles with a maximum stress level of $0.75 \sigma_{\mathrm{Y}}(1000 \mathrm{MPa})$, the type II reaches the same maximum stress but now considering 20 cycles. The type III consists of 10 cycles with a maximum stress level of $0.9 \sigma_{\mathrm{Y}}(1200 \mathrm{MPa})$ and, finally, the scheme IV has a maximum stress of $1200 \mathrm{MPa}$ and 20 cycles.

The four residual stress profiles considered in the present study are classified according to the surface stress state, two of them being of tensile nature and the other two of compressive nature. The parameters defining the profiles, the maximum residual stress at the surface, $\sigma_{\Gamma}$, and the depth $x_{0}$ where the stresses reach a null value, are depicted in Fig. 2. Taking into account this approach the four profiles 1 to 4 are listed in Table 1.

The simulation of the fatigue loading applied to the prestressing steel wire is carried out by the finite element method (FEM) using a commercial code [12]. Due to the axisymmetric geometry of the wire, the three-dimensional (3D) case is simplified to an equivalent two-dimensional (2D) problem. To progress further, the appropriate boundary conditions must be defined, and thus both null values of the normal component of displacements are imposed in symmetry axis and applied load, as sketched in Fig. 3.

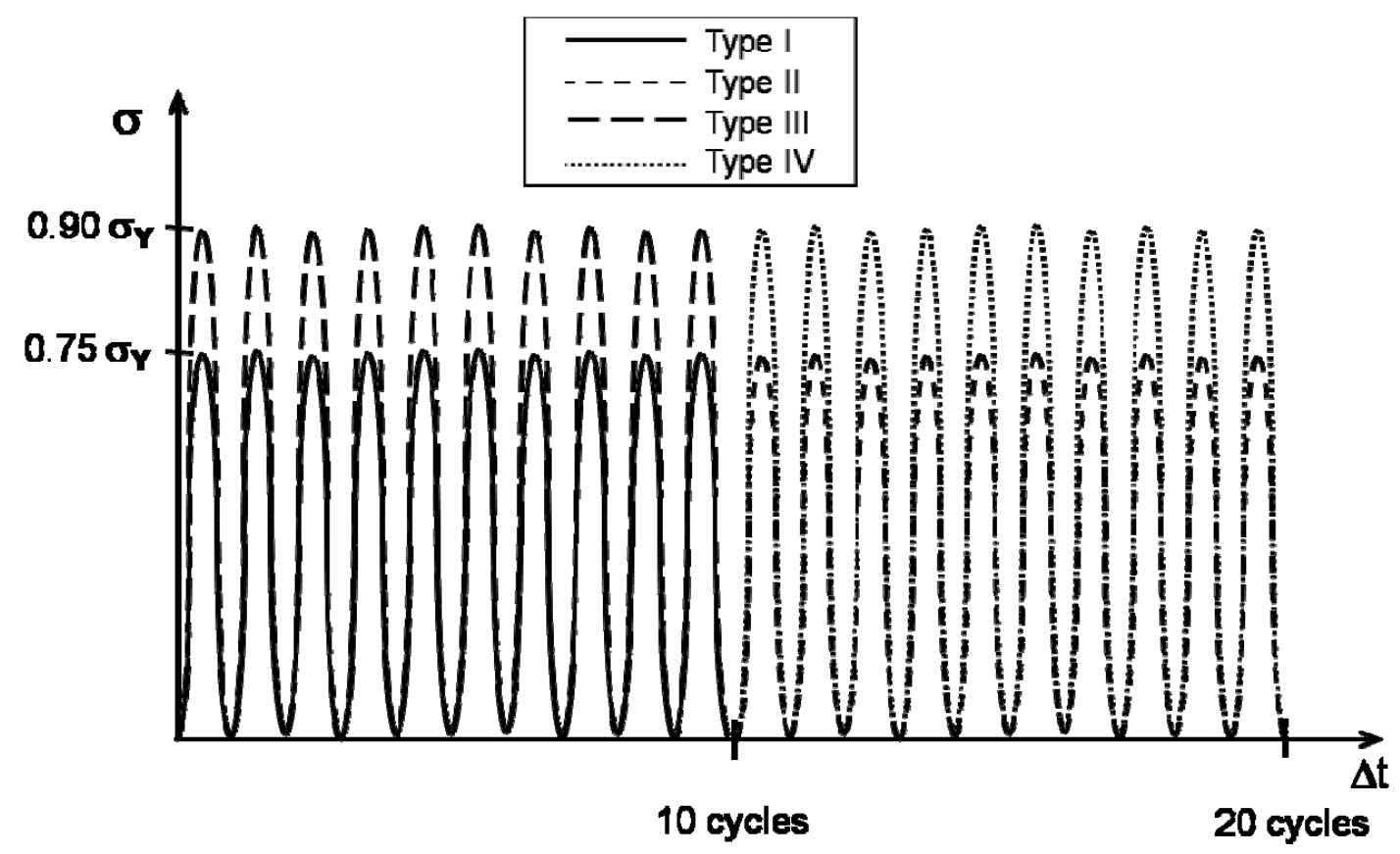

Fig. 1 Fatigue loading schemes applied on the wire: type I (10 cycles, 1000 MPa), type II (20 cycles, 1000 MPa), type III (10 cycles, $1200 \mathrm{MPa}$ ) and type IV (20 cycles, $1200 \mathrm{MPa})$. 


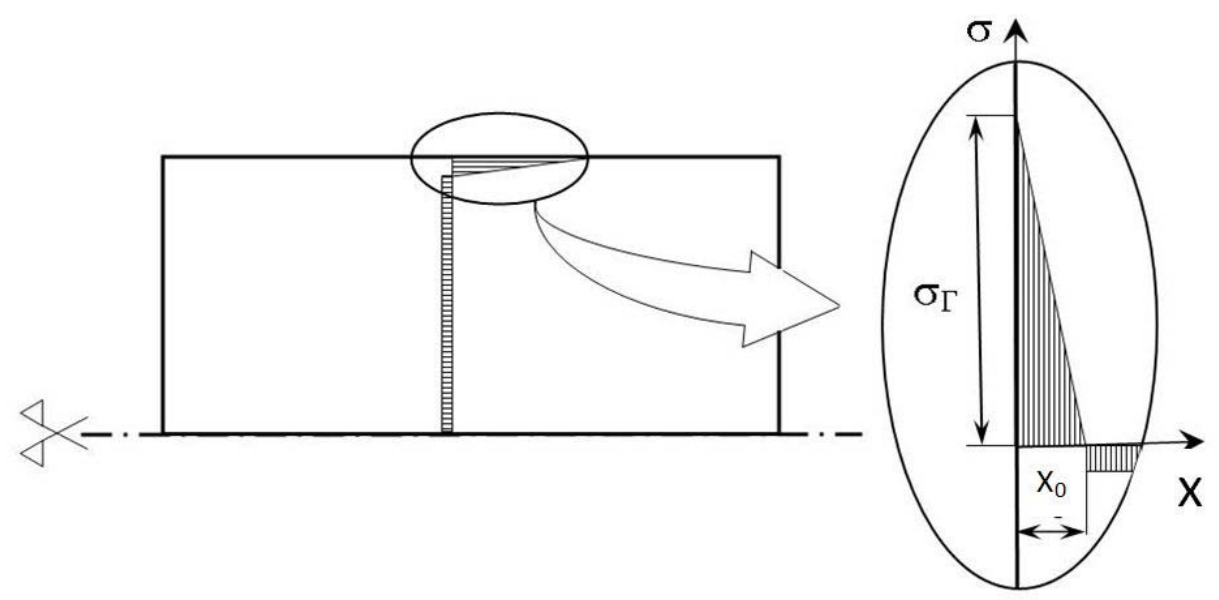

Fig. 2 Scheme of idealized residual stress profile and the parameters needed to define it.

Table 1 Parameters of the ideal residual stress profiles used in the computations.

\begin{tabular}{lcccc}
\hline Profiles & 1 & 2 & 3 & 4 \\
\hline$\sigma_{\Gamma}(\mathrm{MPa})$ & 100 & -100 & 200 & -200 \\
\hline $\mathrm{x}_{0}(\mu \mathrm{m})$ & 250 & 250 & 250 & 250 \\
\hline
\end{tabular}

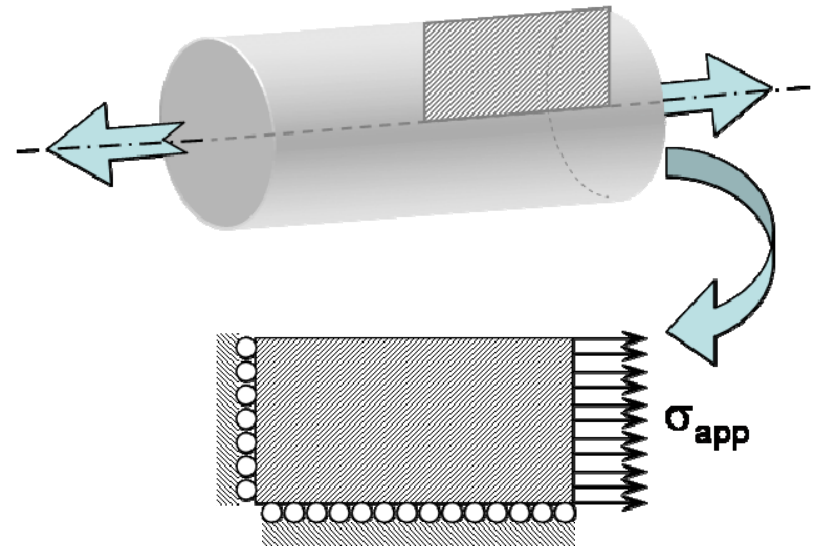

Fig. 3 Specimen and boundary conditions: fixed displacements and externally applied stress $\sigma_{\text {app }}$.

The meshing of the specimen was carried out according to the criterion of reproducing the residual stress profile that has its most significant variations in the vicinity of the wire surface. Consequently, the mesh must be refined in this zone to approximate with more accuracy the profile in the surface. The way to include the residual stress profile is to assign to each element an initial stress value that is dependent on the distance to the wire surface, as shown in Fig. 2. In this sense, Fig. 4 shows this initial stress values from a practical point of view, in comparison with the exact profile previously illustrated.
The meshing of the geometry is developed with four nodes quadrilateral elements due to the simple geometry of the wire axisymetric problem. The simulation is carried out for each residual stress profile with each load. Results of the evolution of the residual stress profile with the loading process are obtained for each type of load.

\section{Influence of the Loading Scheme}

Numerical results are analysed at characteristic instants of the loading history: at initial load, at maximum and minimum load and at final load. Therefore, it is possible to determine the evolution of the initial stress state by comparing the stress states throughout the radius in each one of these loading instants. Although the stress distribution along wire radius at the maximum load instant is obviously higher than at other time instants, it can be assumed that in these ones the stresses are the result of adding the initial residual stress (just after cold drawing) and the externally applied stresses. To compare all time instants, a new parameter called effective stress, $\sigma_{\mathrm{z}}{ }^{*}$, is defined, which is mathematically represented by

$$
\sigma_{\mathrm{z}}^{*} \equiv \sigma_{\mathrm{z}}-\sigma_{\mathrm{app}}
$$

where $\sigma_{z}$ is the axial stress (axial component of the stress tensor) and $\sigma_{\text {app }}$ is the externally applied (remote) stress (cf. Fig. 3). According to the load history reflected in Fig. 1, $\sigma_{\text {app }}$ is null at initial load, minimum 

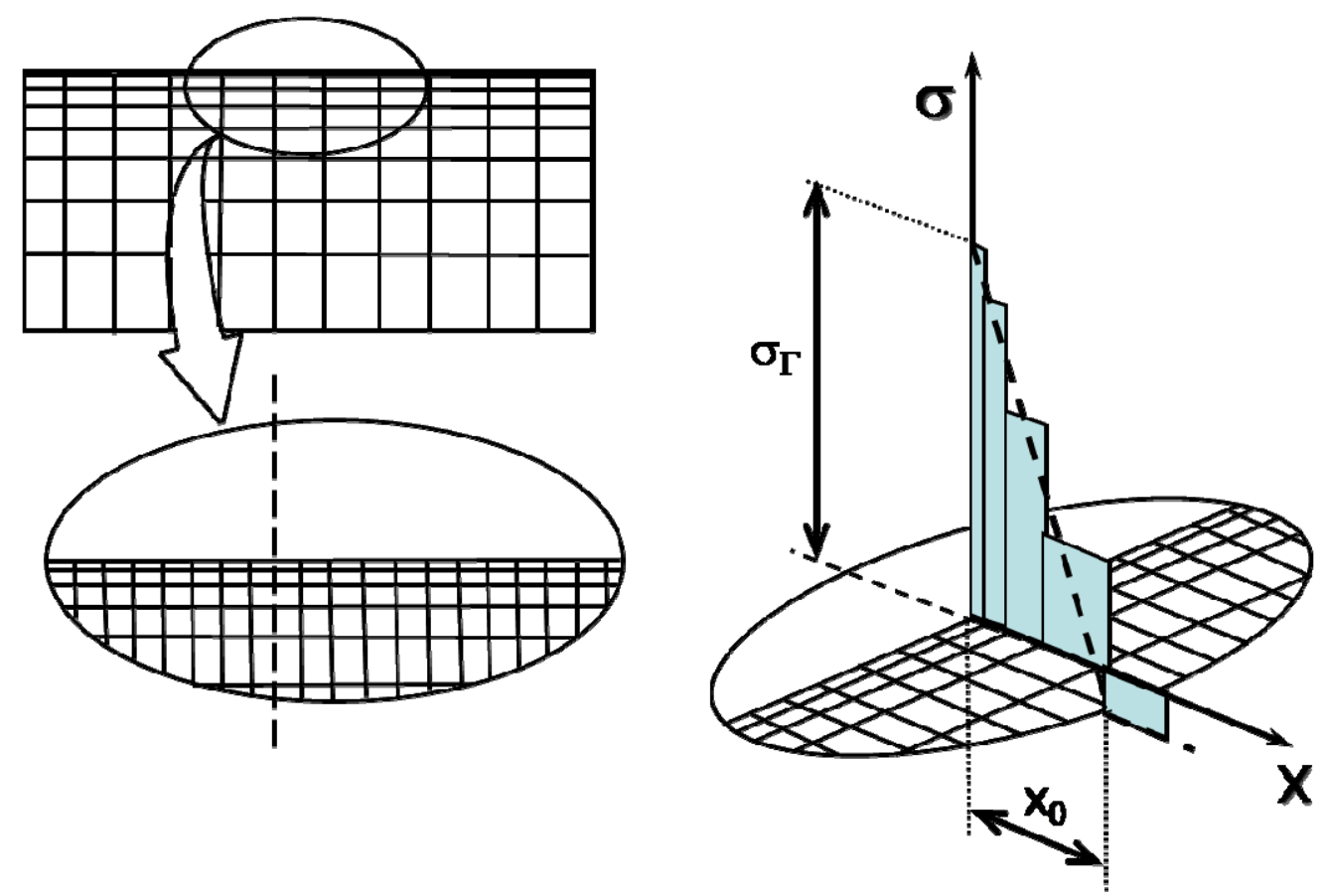

Fig. 4 Meshing of the specimen and detail of the residual stress profile.

load and final load and, consequently, the effective stress is equivalent to the axial stress in this particular cases. On the other hand, at maximum load instants the applied stress is not null and therefore it is possible to consider that $\sigma_{\text {app }}$ acts as a shift factor, thereby bringing this stress distribution near to the other ones.

Taking into account the results obtained in all simulations, stress distributions are stabilized during cycling, so that it is only necessary to represent the last load instant in order to reflect the behaviour of all stress profiles. Fig. 5 compares the effective stress

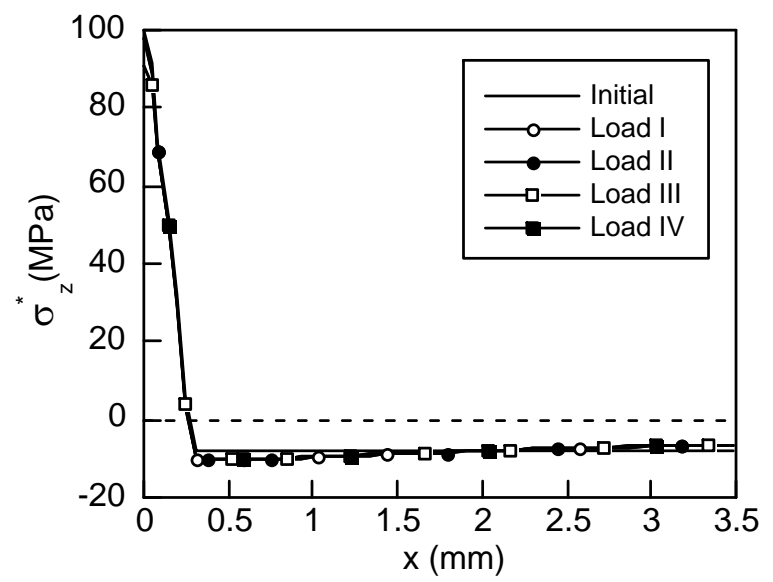

Fig. 5 Effective stress distribution at final loading (residual stress profile 1; loads I-IV). distribution at final load instants, after applying the four loading schemes I to IV to the initial residual stress profile 1.

To study the behaviour of the mean normal (or hydrostatic) stress $\sigma$ (relevant in hydrogen diffusion processes assisted by stresses and strains, and thus in hydrogen embrittlement phenomena) with fatigue loading, a new analysis was developed (Fig. 6), where the same tendency for all applied loads is reflected.

To analyze the stress evolution with time, two different points are considered, one at the outer surface of the material (where the maximum residual stress is achieved) and another placed inside the stable region of the residual stress profile (cf. Fig. 2). The evolutions of mean normal stresses at the three last cycles for a wire with the residual stress profile 1 are shown in Fig. 7 , where $t$ is the dimensionless simulation time. It is observed that the stress evolution follows very closely the load history in both axial and mean normal stresses, as it was expected according to the previous results obtained for the stress profiles at the characteristic instants of the loading process. 


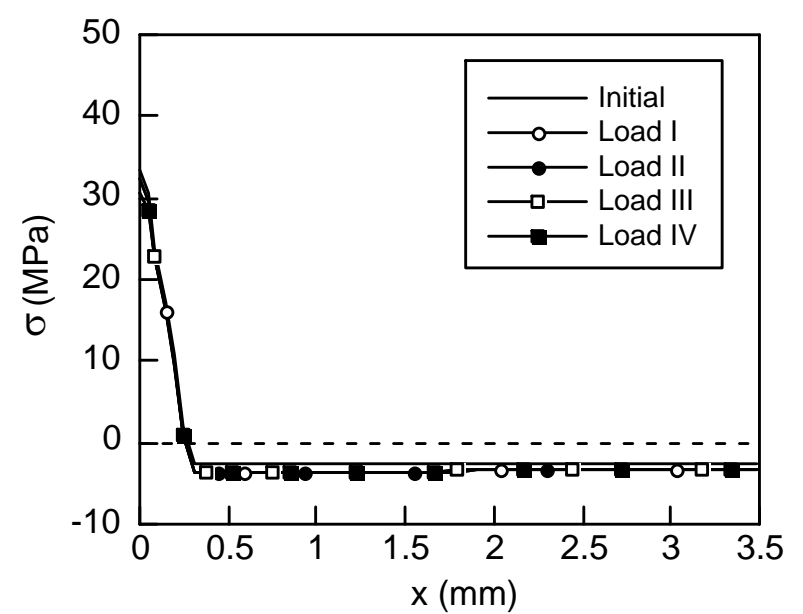

Fig. 6 Hydrostatic stress distribution at final loading (residual stress profile 1; loads I-IV).

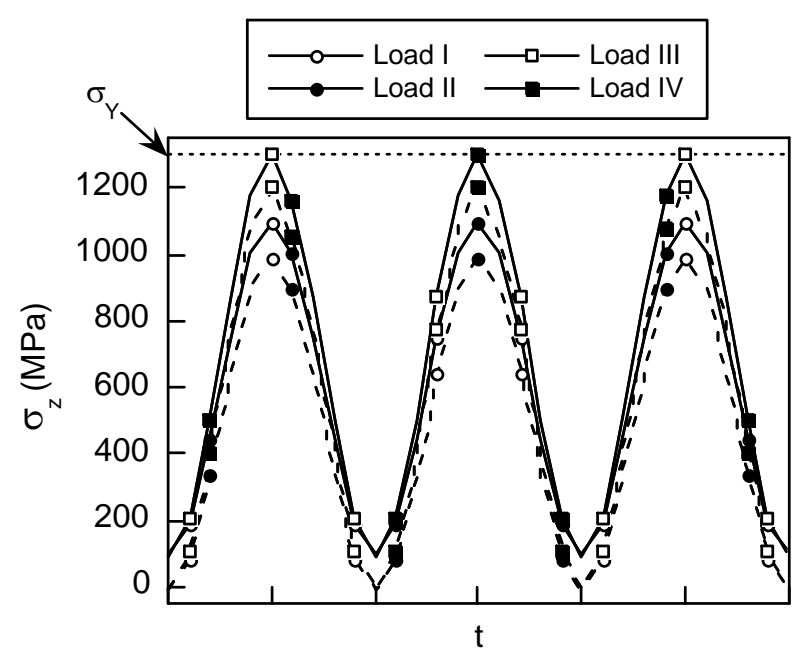

Fig. 7 Axial stress evolution at the three last loading steps for the four loading schemes at external node (solid line) and internal node (dashed line). Residual stress profile 1.

The results obtained for other loads and residual stress profiles are similar to this one. Therefore, it can be seen that fatigue loading does not influence the time evolution of stresses, independently of the considered residual stress profile. Consequently, it is possible to reduce the study because of this analogy between the results for the different fatigue loading schemes and, taking this into account, only the loading schemes I and III are analysed in the next section.

\section{Stress Evolution}

This section deals with the analysis of only two fatigue loading schemes (type I and III, cf. Fig. 1) applied to the four residual stress profiles (cf. Table 1). The obtained results when studying the axial and mean normal stress are equivalent, so that the last one is specially analyzed, due to its more relevant role in stress-assisted diffusion of hydrogen [9-11] and thus on hydrogen embrittlement of materials. As a matter of fact, hydrostatic stress plays a relevant role in the specific problem of hydrogen embrittlement of cold-drawn eutectoid prestressing steels with an associated residual stress state generated during manufacturing process, a process aimed to raise the yield strength and produce a high resistant material to be used in prestressed concrete in civil engineering.

Figs. 8 and 9 show the results obtained with the simulation of the residual stress profiles 1 (100 MPa) and $3(200 \mathrm{MPa})$ respectively, with the maximum stress at surface representing surface tensile stress states and, as it was explained before, only the loading scheme I and III are considered. In these graphs, the characteristic points of the loading history at the specific time instants are represented, namely at initial load, at minimum load, at final load and at maximum load with asterisk, which represents the maximum load with the shift factor $\sigma_{\text {app }}$ applied according to the equation (1). Only minimal differences can be observed between the stresses profiles for the time instants considered in the loading process, although a higher variation is noticed in the profile 3 when the load III is applied. In this case the maximum stress level slightly exceeded the $0 \%$ offset yield strength and, consequently, certain changes in stress distribution are observed.

On the other hand, the analysis of surface compressive stress states, profile 4, is presented in Fig. 10. In this plot no significant differences can be observed regarding the values of mean normal stress at the wire surface for the different characteristic loading instants. The same happened for those tensile residual stresses in which the maximum stress did not exceeded $0 \%$ offset yield stress (a different story happened in those tensile residual stress profiles in which the 


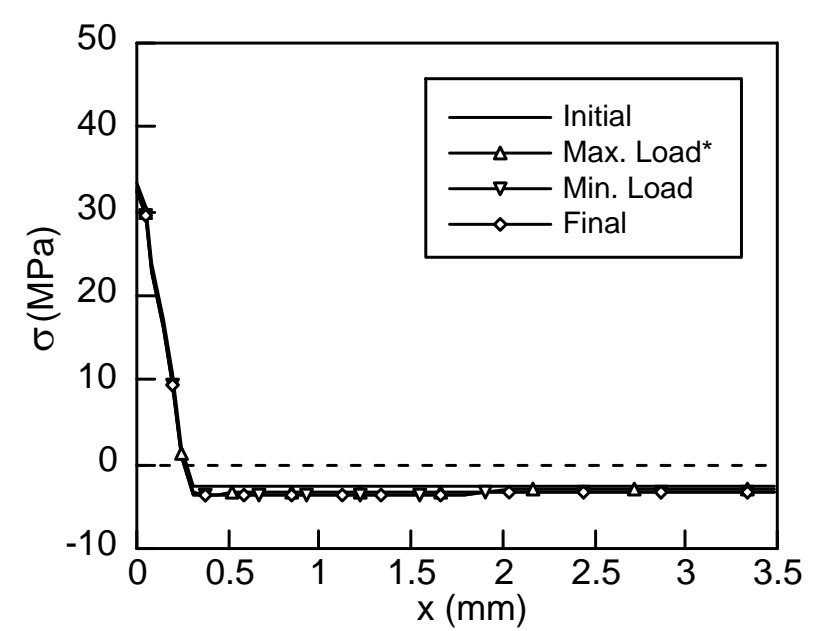

Fig. 8 Hydrostatic stress distribution throughout the wire radius with the residual stress profile 1 at four characteristic instants of the load history $I$.

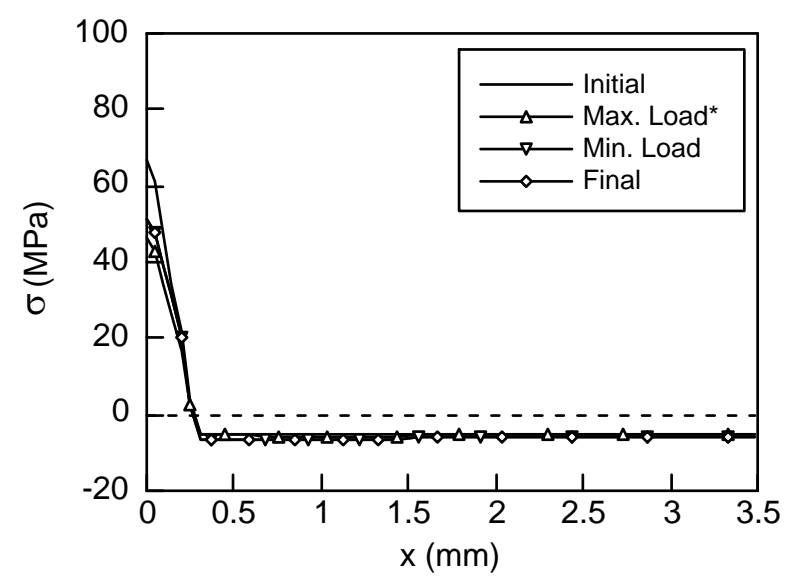

Fig. 9 Hydrostatic stress distribution throughout the wire radius with the residual stress profile 3 at four characteristic instants of the load history III.

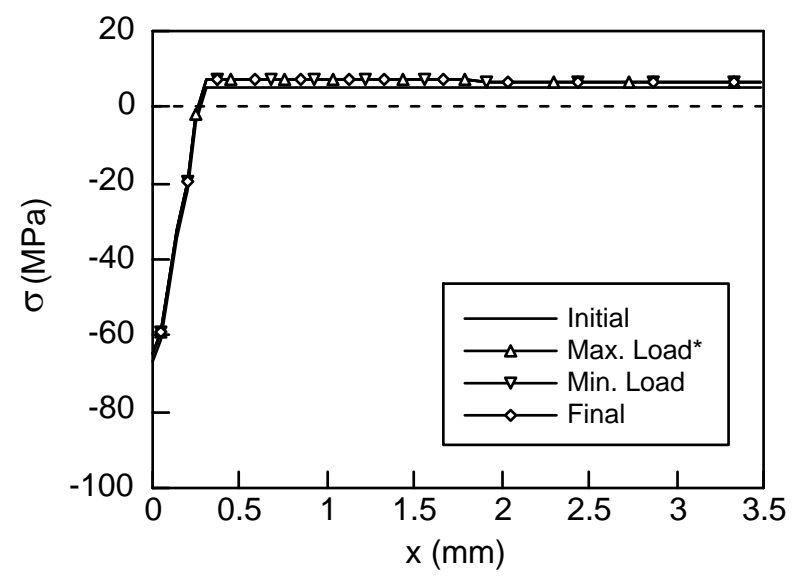

Fig. 10 Hydrostatic stress distribution throughout the wire radius with the residual stress profile 4 at four characteristic instants of the load history $I$. maximum stress level exceeded the $0 \%$ offset yield stress, because in this case some redistribution of residual stress was detected, cf. Fig. 9).

After analysing the plots in Figs. 8-10, it is feasible to point out that the reduction of the stress value at the surface is more pronounced in the residual stress profile 3, probably conditioned by the higher values of boundary stress, $\sigma_{\Gamma}$, thereby affecting the values of mean normal stress at the surface in such profile and conditioning the hydrogen entry in the steel and subsequent embrittlement.

\section{Conclusions}

On the basis of the obtained numerical results, it is concluded that the residual stress profiles studied in this work show no significant changes during the fatigue loading process. The number of cycles of fatigue does not affect the residual stress profile and thus no stress redistribution is observed, but the stress evolution at each node follows the external fatigue loading history wherever it is placed.

The mean normal (hydrostatic) stress exhibits the same behaviour for all the studied fatigue loading schemes at all the time instants. These values are equal to the third part of axial stresses and, consequently, there is a negligible redistribution of the initial state of stresses during the application of the external load.

Finally, it may be concluded that neither the number of cycles nor the maximum load level affect significantly to none of the four residual stress profiles considered in the present paper.

\section{Acknowledgements}

The authors acknowledge the financial support provided by the following Spanish Institutions: MCYT (Grant MAT2002-01831), MEC (Grant BIA2005-08965), MCINN (Grant BIA2008-06810) and JCYL (Grants SA067A05, SA111A07 and SA039A08). 


\section{References}

[1] J. M. Atienza, M. L. Martínez-Pérez, J. Ruiz-Hervias, F. Mompean, M. García-Hernández and M. Elices, Residual stresses in cold drawn ferritic rods, Scripta Materialia 52 (2005) 305-309.

[2] P. J. Webster and G. Mills, Residual stresses in a steel strand, Physica B: Condensed Matter (241-243) (1997) 1270-1273.

[3] H. Sasahara, The effect on fatigue life of residual stress and surface hardness resulting from different cutting conditions of $0.45 \%$ C steel, International Journal of Machine Tools \& Manufacture 45 (2005) 131-136.

[4] Y. M. Xing and J. Lub, An experimental study of residual stress induced by ultrasonic shot peening, Journal of Materials Processing Technology 152 (2004) 56-61.

[5] M. Kuroda, S. Yamanaka and Y. Isobe, Detection of plastic deformation and estimation of maximum value of residual stress in low carbon steel by X-ray stress analysis using statistical techniques, NDT \& E International 36 (2003) 497-502.

[6] J. Toribio, V. Kharin, M. Lorenzo and D. Vergara, Effects of manufacturing-induced residual stresses and strains on hydrogen embrittlement of cold drawn steel, Engineering Structural Integrity: Research, Development and Application (1-2) (2007) 1008-1011.

[7] L. Vehovar, V. Kuhar and A. Vehovar, Hydrogen-assisted stress-corrosion of prestressing wires in a motorway viaduct, Engineering Failure Analysis 5 (1998) 21-27.

[8] J. Woodtli and R. Kieselbach, Damage due to hydrogen embrittlement and stress corrosion cracking, Engineering Failure Analysis 7 (2000) 427-450.

[9] J. Toribio and M. Elices, Influence of residual stresses on hydrogen embrittlement susceptibility of prestressing steels, International Journal of Solid \& Structures 28 (1991) 791-803.

[10] J. Toribio and V. Kharin, A hydrogen diffusion model for applications in fusion nuclear technology, Fusion Engineering and Design (51-52) (2000) 213-218.

[11] J. Toribio, V. Kharin, D. Vergara and M. Lorenzo, Two-dimensional numerical modelling of hydrogen diffusion in metals assisted by both stress and strain, Advanced Materials Research 138 (2010) 117-126.

[12] MARC User Information, Marc Analysis Research Corporation, Palo Alto, 1994. 